\title{
Four-body calculation of elastic deuteron-deuteron scattering
}

\author{
A. Deltuva \\ Institute of Theoretical Physics and Astronomy, Vilnius University, A. Goštauto 12, LT-01108 Vilnius, Lithuania \\ A. C. Fonseca \\ Centro de Física Nuclear da Universidade de Lisboa, P-1649-003 Lisboa, Portugal
}

(Received 29 June, 2015)

\begin{abstract}
Fully converged calculations of deuteron-deuteron elastic scattering observables are performed at energies above three- and four-body breakup threshold. Differential cross sections and analyzing powers are obtained using realistic nucleon-nucleon force models together with the Coulomb repulsion between protons. For all observables we find a very reasonable agreement with the available experimental data limited to deuteron beam energies up to $25.3 \mathrm{MeV}$.
\end{abstract}

PACS numbers: 21.30.-x, 21.45.-v, 24.70.+s, 25.10.+s

\section{INTRODUCTION}

Four-nucleon reactions above breakup threshold pose a highly challenging theoretical and computational problem. In this regime rigorous and realistic results have been obtained so far by only two methodologies. These are the complex-energy method in the framework of momentum-space integral equations [1] and the complex scaling method in the framework of coordinate-space differential equations [2].

In this work we continue our theoretical investigation of the four-nucleon $(4 N)$ scattering above breakup threshold. Previous studies [1, 3 - $[6]$ were mostly devoted to $4 N$ reactions initiated by nucleon-trinucleon $(N+3 N)$ collisions, i.e., neutron $(n)$ or proton $(p)$ beams impinging on ${ }^{3} \mathrm{H}$ or ${ }^{3} \mathrm{He}$ targets. While $n+{ }^{3} \mathrm{H}$ and $p+{ }^{3} \mathrm{He}$ elastic scattering are dominated by states with total $4 N$ isospin $\mathcal{T}=1$, the coupled $n+{ }^{3} \mathrm{He}$ and $p+{ }^{3} \mathrm{H}$ reactions involve both $\mathcal{T}=0$ and $\mathcal{T}=1$. Overall a good reproduction of the experimental data was achieved when using realistic nucleon-nucleon $(N N)$ potentials. The most remarkable discrepancies above breakup threshold are the nucleon analyzing power and polarization, especially for the ${ }^{3} \mathrm{H}(p, n)^{3} \mathrm{He}$ charge exchange reaction, and the minimum of the differential cross section in elastic $p+{ }^{3} \mathrm{He}$ and $n+{ }^{3} \mathrm{He}$ scattering above $25 \mathrm{MeV}$ nucleon energy. One may raise the question whether these disagreements are dominated by $\mathcal{T}=1$ states, or if $\mathcal{T}=0$ components have similar shortcomings as well. The study of deuteron-deuteron $(d+d)$ elastic scattering, the only $4 N$ process dominated by $\mathcal{T}=0$ states, may shed some light on this issue.

Therefore in the present work we concentrate on $d+d$ elastic scattering above breakup threshold. In Ref. 7] we already calculated $d+d$ reactions around $E_{d}=10 \mathrm{MeV}$ deuteron beam energy and found quite a good agreement with data, but also some inconsistencies between different data sets, thereby calling for a more extensive study over a wider energy range. Here we present results at $E_{d}$ ranging from $4.75 \mathrm{MeV}$, just slightly above the threecluster threshold of $4.45 \mathrm{MeV}$, to $25.3 \mathrm{MeV}$. Restricting the model space to $4 N$ states with $\mathcal{T}=0$ precludes to obtain simultaneously reliable amplitudes for ${ }^{2} \mathrm{H}(d, p)^{3} \mathrm{H}$ and ${ }^{2} \mathrm{H}(d, n)^{3} \mathrm{He}$ transfer reactions, but has an important practical advantage, namely, allows for the reduction of the number of basis states needed to get convergence for the $d+d$ elastic observables and thereby speeds up the calculations.

In Sec. II we present the theoretical framework and the reliability of using $\mathcal{T}=0$ states alone to calculate $d+d$ elastic scattering. Differential cross section and analyzing power results are shown in Sec. III and a summary is presented in Sec. IV.

\section{THEORY}

The four-particle collision process is described by exact Alt, Grassberger, and Sandhas (AGS) equations [8, 9] for the transition operators $\mathcal{U}_{\beta \alpha}$ whose components are labeled according to the chains of partitions. Given that neutrons and protons in the isospin formalism are treated as identical particles, there are only two chains of partitions that can be distinguished by the two-cluster partitions, one $(\alpha=1)$ being of the $3+1$ type, i.e., (12,3)4, and another $(\alpha=2)$ being of the $2+2$ type, i.e., (12)(34). For the nucleon-trinucleon scattering in previous works we solved the symmetrized AGS equations for $\mathcal{U}_{\beta 1}$ but the reactions initiated by two deuterons require transition operators $\mathcal{U}_{\beta 2}$. In both cases the AGS equations share the same kernel but differ in the driving term. Thus, in the present work we solve the integral equations

$$
\begin{aligned}
& \mathcal{U}_{12}=\left(G_{0} t G_{0}\right)^{-1}-P_{34} U_{1} G_{0} t G_{0} \mathcal{U}_{12}+U_{2} G_{0} t G_{0} \mathcal{U}_{22} \\
& \mathcal{U}_{22}=\left(1-P_{34}\right) U_{1} G_{0} t G_{0} \mathcal{U}_{12}
\end{aligned}
$$

Here $t$ is the two-nucleon transition matrix, $U_{1}$ and $U_{2}$ are the transition operators for the $1+3$ and $2+2$ subsystems, $P_{34}$ is the permutation operator of particles 3 and 4 , and $G_{0}=\left(E+i \varepsilon-H_{0}\right)^{-1}$ is the free four-particle resolvent at the available energy $E$, whereas $H_{0}$ is the free Hamiltonian. Although the physical scattering pro- 


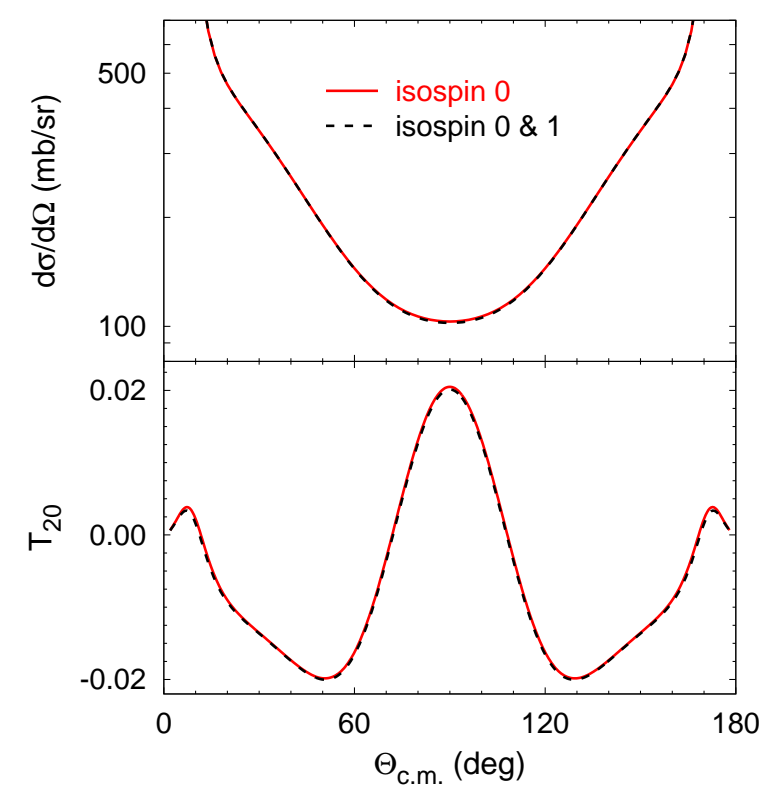

FIG. 1. (Color online) Deuteron-deuteron elastic scattering at $10 \mathrm{MeV}$ beam energy. Differential cross section and deuteron tensor analyzing power $T_{20}$ calculated neglecting (solid curves) and including (dashed curves) total isospin $\mathcal{T}=1$ states are compared.

cess corresponds to $\varepsilon \rightarrow+0$, the complex energy method uses finite $\varepsilon$ value when solving the AGS equations numerically. The physical scattering amplitudes are then obtained by extrapolating finite $\varepsilon$ results to the $\varepsilon \rightarrow+0$ limit. The extrapolation procedure as well as the special method for integrals with quasi-singularities encountered when solving Eqs. (11) are described in detail in our previous works [1, 5, 10].

The asymptotic $d+d$ channel state is a pure total isospin $\mathcal{T}=0$ state. However, due to the charge dependence of the $N N$ interaction the scattering equations (11) couple the states with different $\mathcal{T}$. Nevertheless, the effect of $\mathcal{T}>0$ states on the $d+d$ elastic scattering is of second order in the charge-dependent terms of the $N N$ interaction that are dominated by the $p p$ Coulomb force; a smaller contribution from the hadronic part is present as well. Therefore at not too low energies one may expect these effects to be small. This conjecture is well supported by our test calculations for $d+d$ elastic scattering where we find a tiny effect of the $\mathcal{T}=1$ states, as presented in Fig. 1 for the differential cross section $d \sigma / d \Omega$ and tensor analyzing power $T_{20}$, both as functions of the center-of-mass (c.m.) scattering angle $\Theta_{\text {c.m. }}$. The results in the next section therefore are obtained by solving Eqs. (1) with $\mathcal{T}=0$ states alone. This reduces the number of basis states by more than a factor of 2 thereby speeding up the practical calculations significantly. With $\mathcal{T}=0$ the isospin of the $3 N$ subsystem is limited to $T_{y}=\frac{1}{2}$; the needed isospin components of the two-nucleon transition matrix $t$ are given in Ref. [5].)
The $p p$ Coulomb force is included using the method of screening and renormalization [11, 12] where the screening radius $R=10$ to $12 \mathrm{fm}$ is found to be sufficient to get convergence for the Coulomb-distorted short-range part of the amplitude. The obtained results are well converged with respect to the partial-wave expansion. When solving Eqs. (1) we take into account isospin-singlet $2 N$ partial waves with total angular momentum $j_{x} \leq 4$ and isospin-triplet $2 \mathrm{~N}$ partial waves with orbital angular momentum $l_{x} \leq 7,3 \mathrm{~N}$ partial waves with spectator orbital angular momentum $l_{y} \leq 7$ and total angular momentum $J \leq \frac{13}{2}$, and $4 N$ partial waves with $1+3$ and $2+2$ orbital angular momentum $l_{z} \leq 7$. Initial and final deuterondeuteron states with relative orbital angular momentum $L \leq 4$ are sufficient for the calculation of observables except at the $25.3 \mathrm{MeV}$ beam energy where we take into account also the states up to $L \leq 6$ yielding a small but visible contribution.

\section{RESULTS}

The scattering of two deuterons is both challenging from the computational point of view and interesting visa-vis nucleon-trinucleon scattering. Since deuterons are loosely bound and spatially large objects, their collision gives rise to much higher breakup cross sections than encountered in other $4 N$ reactions initiated by either neutrons or protons.

\begin{tabular}{lcc}
\hline \hline & $B\left({ }^{3} \mathrm{H}\right)$ & $B\left({ }^{3} \mathrm{He}\right)$ \\
\hline N3LO & 7.85 & 7.13 \\
CD Bonn & 8.00 & 7.26 \\
CD Bonn $+\Delta$ & 8.28 & 7.53 \\
INOY04 & 8.49 & 7.73 \\
Experiment & 8.48 & 7.72 \\
\hline \hline
\end{tabular}

TABLE I. ${ }^{3} \mathrm{H}$ and ${ }^{3} \mathrm{He}$ binding energies (in $\mathrm{MeV}$ ) for different $N N$ potentials.

We calculate differential cross section and deuteron analyzing powers for $d+d$ elastic scattering at deuteron beam energies $E_{d}$ ranging from 4.75 to $25.3 \mathrm{MeV}$. Given the identity of the deuterons, the observables are either symmetric or antisymmetric with respect to the centerof-mass scattering angle $\Theta_{\text {c.m. }}=90^{\circ}$. At all considered energies the results are obtained using the realistic inside-nonlocal outside-Yukawa (INOY04) potential by Doleschall [13, 14]. It nearly reproduces the experimental values of ${ }^{3} \mathrm{He}$ and ${ }^{3} \mathrm{H}$ binding energy without an additional $3 N$ force. To investigate the dependence of the results on the interaction model, at several energies, i.e., $E_{d}=6,10,11.5,11.57$, and $25.3 \mathrm{MeV}$ we show also the predictions obtained with other high-precision $N N$ potentials. These are the chiral effective field theory potential at next-to-next-to-next-to-leading order (N3LO) [15], the charge-dependent Bonn potential (CD Bonn) 


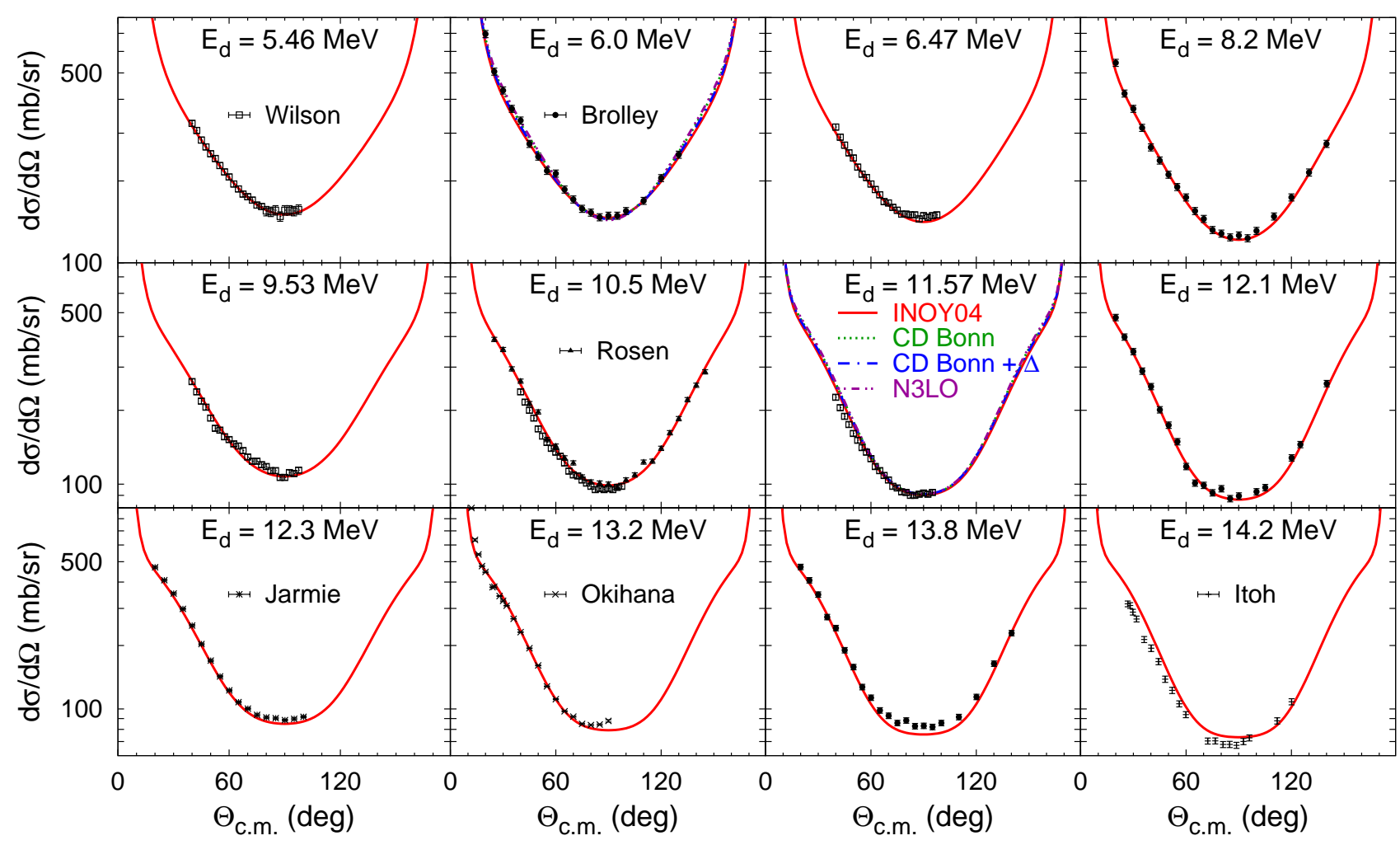

FIG. 2. (Color online) Differential cross section of $d+d$ elastic scattering as a function of c.m. scattering angle at deuteron beam energies ranging from 5.46 to $14.2 \mathrm{MeV}$. Results are obtained using INOY04 potential (solid curves), and, at 6.0 and 11.57 MeV, also CD Bonn $+\Delta$ (dashed-dotted curves), CD Bonn (dotted curves), and N3LO (double-dotted-dashed curves) potentials. The experimental data are from Refs. [19 24].

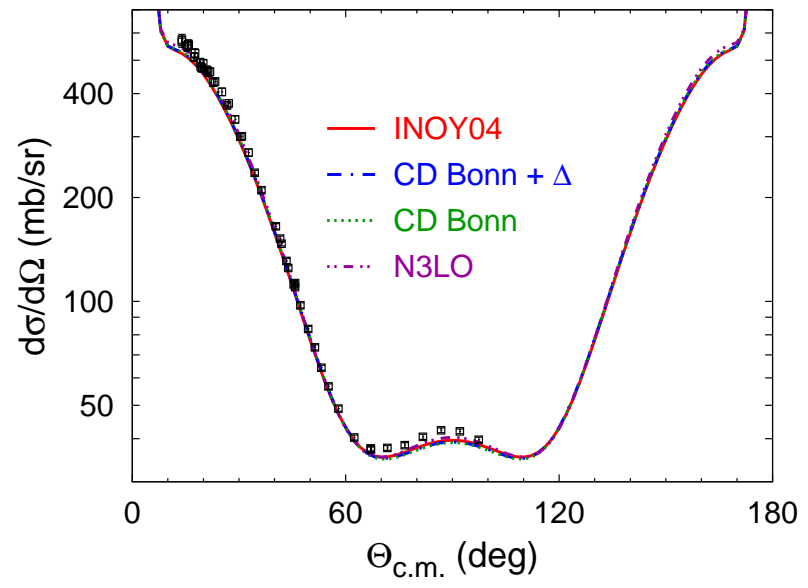

FIG. 3. (Color online) Differential cross section of $d+d$ elastic scattering at $E_{d}=25.3 \mathrm{MeV}$. Curves are as in Fig. 2, The experimental data are from Ref. [25].

[16], and its extension CD Bonn $+\Delta$ [17] explicitly including an excitation of a nucleon to a $\Delta$ isobar. This mechanism generates effective $3 N$ and $4 N$ forces that are mutually consistent but quantitatively still insufficient to reproduce $3 N$ and $4 N$ binding energies, although they reduce the discrepancy [18]. The predictions of ${ }^{3} \mathrm{He}$ and ${ }^{3} \mathrm{H}$ binding energy for all employed force models are collected in Table 【

In Fig. 2 we show results for the $d+d$ elastic differential cross section in the $E_{d}$ range between 5.46 and 14.2 $\mathrm{MeV}$. In this regime $d \sigma / d \Omega$ shows quite a simple angular dependence, having forward and backward peaks where $d \sigma / d \Omega$ becomes infinite due to the Coulomb force and a single local minimum at $\Theta_{\text {c.m. }}=90^{\circ}$. The calculations describe the data [19 22] well up to $E_{d}=12.1 \mathrm{MeV}$ and show little sensitivity to the $N N$ force model as one may naively expect given the large size of both projectile and target nuclei. The largest observed difference between the four employed force models amounts about $6 \%$ at $E_{d}=6 \mathrm{MeV}$ around $\Theta_{\text {c.m. }}=40^{\circ}$ and $140^{\circ}$. For $E_{d}$ above $12.3 \mathrm{MeV}$ small discrepancies between data and calculations emerge, mostly around $\Theta_{\text {c.m. }}=90^{\circ}$. However, one probably should question the quality of the data [23] near $\Theta_{\text {c.m. }}=90^{\circ}$ where, in contrast to all other sets, a local maximum is present, as well as the quality of the data 24] that lacks symmetry with respect to $\Theta_{\text {c.m. }}=90^{\circ}$ and seems to be inconsistent with a smooth energy-dependence seen in theoretical predictions and other data sets. Our calculations overpredict 


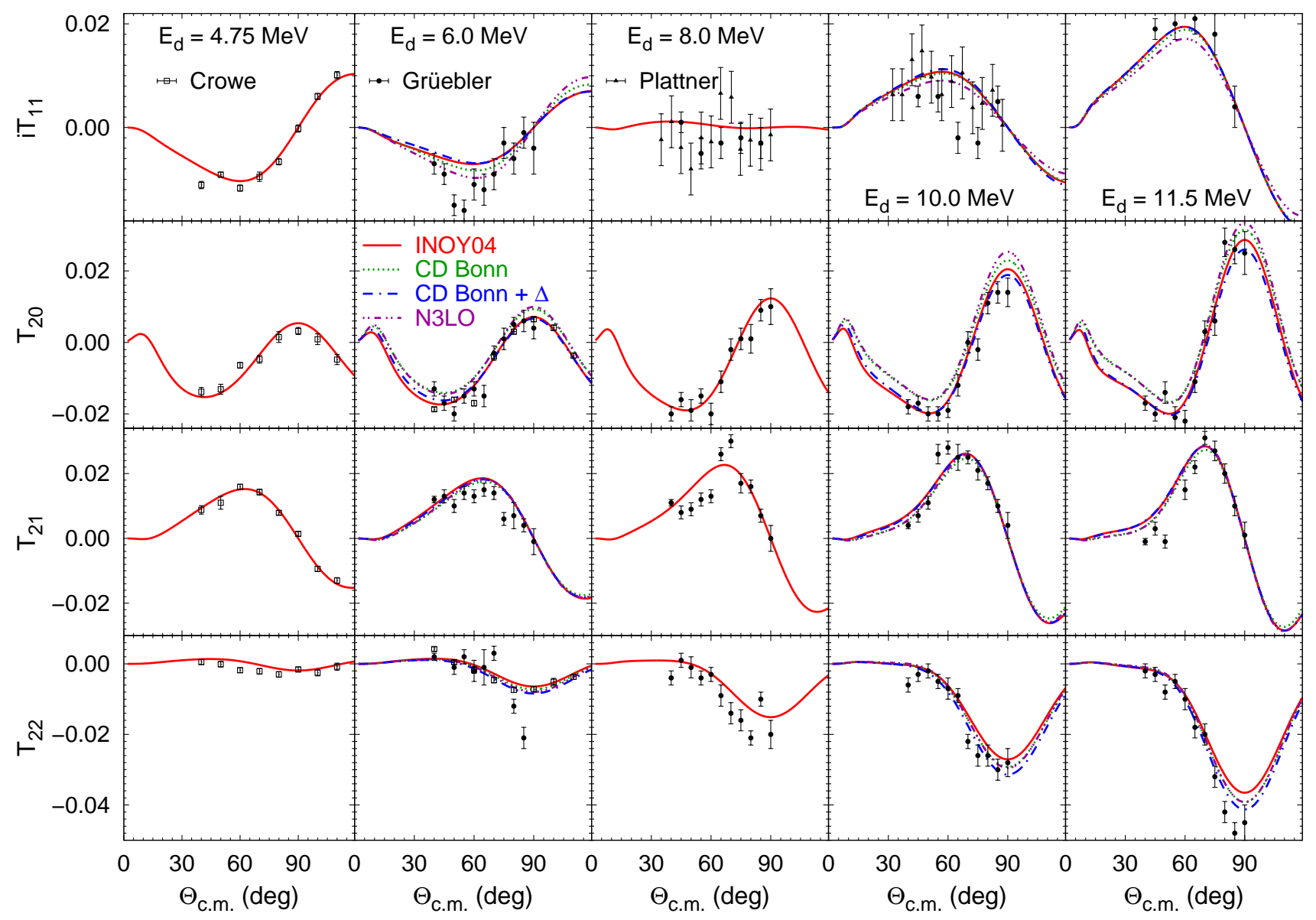

FIG. 4. (Color online) Deuteron analyzing powers in $d+d$ elastic scattering at $E_{d}=4.75,6.0,8.0,10.0$, and $11.5 \mathrm{MeV}$. Curves are as in Fig. 20 The experimental data are from Refs. 26 28].
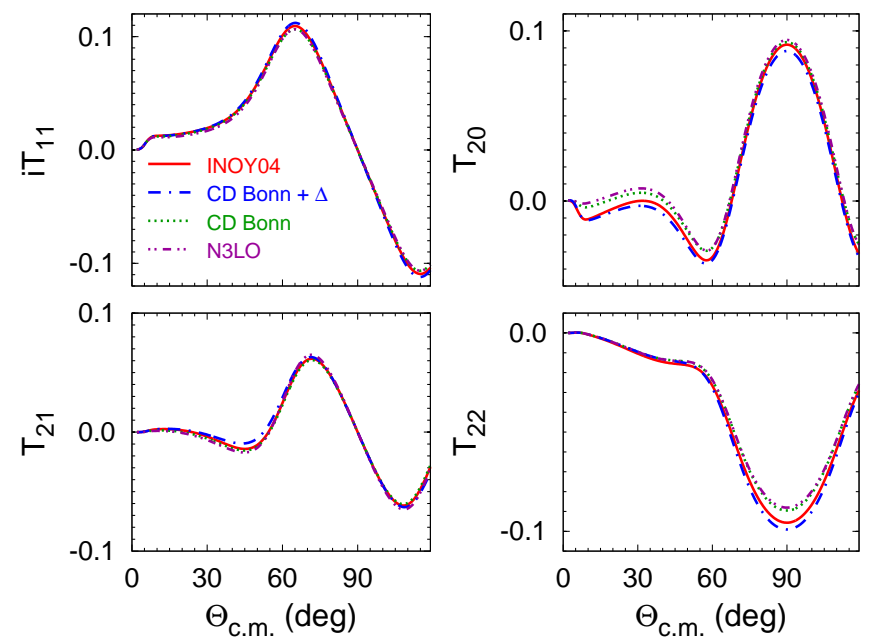

FIG. 5. (Color online) Deuteron analyzing powers in $d+d$ elastic scattering at $E_{d}=25.3 \mathrm{MeV}$. Curves are as in Fig. 2 the $14.2 \mathrm{MeV}$ data 24 by up to $9 \%$ but underpredict the $13.8 \mathrm{MeV}$ data 20] around the minimum by the same amount. Unfortunately, the available experimental data are very scarce above $E_{d}=15 \mathrm{MeV}$. To the best of our knowledge, up to $E_{d}=35 \mathrm{MeV}$ which is the reach of the present calculations there is only one reliable data set at $E_{d}=25.3 \mathrm{MeV}$ [25]. As shown in Fig. 3, at this higher energy the differential cross section finally develops a more complicated angular dependence with a local maximum at $\Theta_{\text {c.m. }}=90^{\circ}$ and two local minima around $\Theta_{\text {c.m. }}=70^{\circ}$ and $110^{\circ}$. The calculations reproduce well the shape of the data but slightly underpredict its magnitude by about $6 \%$ at forward angles and around $\Theta_{\text {c.m. }}=90^{\circ}$. Although the outlook is somehow contradictory, it looks like the calculations slightly underpredict the experimental $d \sigma / d \Omega$ data above $E_{d}=13 \mathrm{MeV}$. In contrast to lower energies where the minimum region is insensitive to the $N N$ potential, at $E_{d}=25.3 \mathrm{MeV}$ at $\Theta_{\text {c.m. }}=90^{\circ}$ the spread of predictions is about $3 \%$ but shows no correlation with $3 N$ binding energy.

In Fig. 4 we present the vector analyzing power $i T_{11}$ and tensor analyzing powers $T_{20}, T_{21}$, and $T_{22}$ for $E_{d}=$ $4.75,6.0,8.0,10.0$, and $11.5 \mathrm{MeV}$. Given the symmetry 
$\left(T_{20}, T_{22}\right)$ or antisymmetry $\left(i T_{11}, T_{21}\right)$ of these observables with respect to $\Theta_{\text {c.m. }}=90^{\circ}$ and the absence of the data for backward angles, we show only the regime up to $\Theta_{\text {c.m. }}=120^{\circ}$. The overall agreement between theoretical predictions and experimental data is good. Due to the large spatial size of the deuteron, all these spin observables are very small in their magnitude, in a sharp contrast with reactions involving initial and/or final nucleontrinucleon states. Unlike the differential cross section, the analyzing powers exhibit not only a more complex behavior as functions of energy and scattering angle, but also a greater sensitivity to the used $N N$ force model, especially for $T_{20}$ and around the extrema of $i T_{11}$ and $T_{22}$. While tensor analyzing powers monotonically increase in their magnitude with moderate changes in the shape as the energy increases, the vector analyzing power $i T_{11}$ exhibits a change of sign after nearly vanishing at $E_{d}=8.0 \mathrm{MeV}$. The error bars exceed the force model dependence for $i T_{11}$ and $T_{21}$. Predictions based on N3LO and CD Bonn potentials slightly deviate from the data for $T_{20}$ above $10 \mathrm{MeV}$ whereas INOY04 and CD Bonn $+\Delta$ reproduce the data well. However, INOY04 slightly underestimates the magnitude of $T_{22}$ whereas CD Bonn $+\Delta$ provides the best description of this observable. In general, the dependence of the observables on the details of the interaction is more complicated than just a simple scaling with the trinucleon binding energy or deuteron $D$-state probability. The $\Delta$-isobar excitation appears to be more important than in the nucleon-trinucleon scattering.

Although there is no data available for polarization observables at $E_{d}=25.3 \mathrm{MeV}$, we present them in Fig. 5 to demonstrate the increase in their magnitude and the development of a more complicated angular dependence.
However, the relative sensitivity to the $N N$ force model is not increased.

\section{SUMMARY}

In the present manuscript we solve the four-body AGS equations for $d+d$ elastic scattering over a wide energy range above breakup threshold using realistic force models between nucleons that are based on either chiral effective field theory or meson exchange theory. The Coulomb interaction between protons is included through the method of screening and renormalization. The calculations are fully converged in terms of partial wave expansion and discretization grids for the momentum variables. In these calculation we have only included total isospin $\mathcal{T}=0$ states alone, given that $\mathcal{T}=1$ states have an extremely small contribution to $d+d$ elastic scattering, as demonstrated in Sec. II. Overall, no striking disagreement with the data is observed. The calculated observables follow the energy trend of the experimental data up to $E_{d}=25.3 \mathrm{MeV}$, which is the maximum energy we have calculated at this time. We find that there is a slight underprediction of the differential cross section minimum beyond $13.2 \mathrm{MeV}$; however, this is not in a full analogy with the $p+{ }^{3} \mathrm{He}$ and $n+{ }^{3} \mathrm{He}$ elastic scattering since the discrepancy in $d+d$ neither increases with the beam energy from 13.8 to $25.3 \mathrm{MeV}$ nor scales with $3 N$ binding energy. We also observe no increase in sensitivity for spin observables compared to what is found in $N+3 N$ scattering. In contrast to $N+3 N$ and $p+d$ collisions, the vector analyzing power in $d+d$ elastic scattering is described quite well.
[1] A. Deltuva and A. C. Fonseca, Phys. Rev. C 86, 011001(R) (2012).

[2] R. Lazauskas, Phys. Rev. C 91, 041001 (2015).

[3] A. Deltuva and A. C. Fonseca, Phys. Rev. C 87, 054002 (2013).

[4] A. Deltuva and A. C. Fonseca, Phys. Rev. Lett. 113, 102502 (2014).

[5] A. Deltuva and A. C. Fonseca, Phys. Rev. C. 90, 044002 (2014).

[6] A. Deltuva and A. C. Fonseca, Phys. Rev. C 91, 034001 (2015).

[7] A. Deltuva and A. Fonseca, Phys. Lett. B 742, 285 (2015).

[8] E. O. Alt, P. Grassberger, and W. Sandhas, JINR report E4-6688, 1 (1972).

[9] P. Grassberger and W. Sandhas, Nucl. Phys. B2, 181 (1967).

[10] J. Carbonell, A. Deltuva, A. Fonseca, and R. Lazauskas, Progress in Particle and Nuclear Physics 74, 55 (2014).

[11] E. O. Alt and W. Sandhas, Phys. Rev. C 21, 1733 (1980).

[12] A. Deltuva and A. C. Fonseca, Phys. Rev. Lett. 98, 162502 (2007).

[13] P. Doleschall, Phys. Rev. C 69, 054001 (2004).
[14] R. Lazauskas and J. Carbonell, Phys. Rev. C 70, 044002 (2004).

[15] D. R. Entem and R. Machleidt, Phys. Rev. C 68, 041001(R) (2003).

[16] R. Machleidt, Phys. Rev. C 63, 024001 (2001).

[17] A. Deltuva, R. Machleidt, and P. U. Sauer, Phys. Rev. C 68, 024005 (2003).

[18] A. Deltuva, A. C. Fonseca, and P. U. Sauer, Phys. Lett. B 660, 471 (2008).

[19] A. Wilson, M. Taylor, J. Legg, and G. Phillips, Nucl. Phys. A 126, 193 (1969).

[20] J. E. Brolley, T. M. Putnam, L. Rosen, and L. Stewart, Phys. Rev. 117, 1307 (1960).

[21] L. Rosen and J. C. Allred, Phys. Rev. 88, 431 (1952).

[22] N. Jarmie and J. H. Jett, Phys. Rev. C 10, 54 (1974).

[23] A. Okihana et al, Jap. Phys. J. 46, 707 (1979).

[24] H. Itoh, Prog. Theor. Phys. 39, 1361 (1968).

[25] W. T. H. Van Oers, H. Arnold, and K. W. Brockman Jr., Nucl. Phys. 46, 611 (1963).

[26] B. J. Crowe, C. R. Brune, W. H. Geist, H. J. Karwowski, E. J. Ludwig, K. D. Veal, A. C. Fonseca, G. M. Hale, and K. A. Fletcher, Phys. Rev. C 61, 034006 (2000). 
[27] W. Grüebler, V. König, R. Risler, P. Schmelzbach, R. White, and P. Marmier, Nucl. Phys. A 193, 149 (1972).

[28] G. R. Plattner and L. G. Keller, Phys. Lett. B 30, 327 (1969). 